\title{
The Harary Index of All Unicyclic Graphs with Given Diameter
}

\author{
Bao-Hua Xing, ${ }^{1}$ Gui-Dong Yu $\mathbb{D},{ }^{1,2}$ Li-Xiang Wang, ${ }^{1}$ and Jinde Cao $\mathbb{D}^{3}$ \\ ${ }^{1}$ School of Mathematics and Computation Sciences, Anqing Normal University, Anqing 246133, China \\ ${ }^{2}$ Basic Department, Hefei Preschool Education College, Hefei 230013, China \\ ${ }^{3}$ School of Mathematics, Southeast University, Nanjing 210096, China
}

Correspondence should be addressed to Jinde Cao; jdcao@seu.edu.cn

Received 15 June 2018; Revised 23 July 2018; Accepted 7 August 2018; Published 16 September 2018

Academic Editor: Beatrice Di Bella

Copyright (C) 2018 Bao-Hua Xing et al. This is an open access article distributed under the Creative Commons Attribution License, which permits unrestricted use, distribution, and reproduction in any medium, provided the original work is properly cited.

The Harary index of $G$ is the sum of reciprocals of distance between any two vertices in $G$. In this paper, we obtain the graphs with the maximum and second-maximum Harary indices among $n$-vertex unicyclic graphs with diameter $d$.

\section{Introduction}

In this paper, all graphs are simple, undirected, and connected. Denote by $E\left(G_{1}\right), V\left(G_{1}\right)$ the edge set and vertex set of $G_{1}$ and $\left|E\left(G_{1}\right)\right|,\left|V\left(G_{1}\right)\right|$ the number of edges and vertices in $G_{1}$, respectively. When $\left|E\left(G_{1}\right)\right|=\left|V\left(G_{1}\right)\right|$, the graph $G_{1}$ is called a unicyclic graph. Let $d(x)$ (or $\left.d_{G_{1}}(x)\right), N_{G_{1}}(x)$ be the degree and the neighborhood of a vertex $x$ in $G_{1}$, respectively. When $d(x)=1$, we call $x$ is a pendant vertex. The distance between $x_{1}$ and $y_{1}\left(x_{1}, y_{1} \in V\left(G_{1}\right)\right)$ is denoted by $d_{G_{1}}\left(x_{1}, y_{1}\right)$. The diameter $d$ of $G_{1}$ is $d=\max \left\{d_{G_{1}}\left(x_{1}, y_{1}\right) \mid \forall x_{1}, y_{1} \in\right.$ $\left.V\left(G_{1}\right)\right\}$. The graph $G_{1}-x_{1} y_{1}$ (or $\left.G_{1}+x_{1} y_{1}\right)$ arisen by deleting the edge $x_{1} y_{1} \in E\left(G_{1}\right)$ (or by adding a new edge $x_{1} y_{1} \notin$ $\left.E\left(G_{1}\right)\right)$. Let $P_{k}$ and $C_{k}$ be the path and cycle with $\left|V\left(P_{k}\right)\right|=$ $\left|V\left(C_{k}\right)\right|=k$, respectively. Denote by $P=v_{0} v_{1} \cdots v_{k}(k \geq 1)$ a path of $G_{1}$, where $d_{G_{1}}\left(v_{1}\right)=d_{G_{1}}\left(v_{2}\right)=\cdots=d_{G_{1}}\left(v_{k-1}\right)=2$ (if $k>1$ ). When $d_{G_{1}}\left(v_{0}\right) \geq 3, d_{G_{1}}\left(v_{k}\right) \geq 3, P$ is an internal path of $G_{1}$. When $d_{G_{1}}\left(v_{0}\right) \geq 3, d_{G_{1}}\left(v_{k}\right)=1, P$ is a pendant path of $G_{1}$. If $k=1$, specially, $P$ is a pendant edge. When the subgraph $P_{1}$ is $P$ itself, where $P_{1}$ is induced by $V(P)$ in $G_{1}, P$ is an induced path. For other terminologies and notations, we refer the readers to [1].

The Harary index $H\left(G_{1}\right)$, has been introduced independently in $[2,3]$. Its calculation is as follows:

$$
H\left(G_{1}\right)=\sum_{x_{1}, y_{1} \in V\left(G_{1}\right)} \frac{1}{d_{G_{1}}\left(x_{1}, y_{1}\right)},
$$

where $d_{G_{1}}\left(x_{1}, y_{1}\right)$ is defined as above and the sum goes over all the pairs of vertices in $G_{1}$.
The set of $n$-vertex unicyclic graphs with diameter $d$ is denoted by $U_{n, d}$. The graph $\triangle_{n, d}^{1}$ (see Figure 1 ) on $n$ vertices arisen from $P_{d}=v_{0} v_{1} \cdots v_{d}$ by attaching $n-d-2$ pendant edges to $v_{\lfloor d / 2\rfloor}$ and adding a new vertex $v_{d+1}$ to be adjacent to $v_{\lfloor d / 2\rfloor}$ and $v_{\lfloor d / 2\rfloor+1}$.

The graph $\triangle_{n, d}^{2}$ (see Figure 1) arisen from $P_{d}=v_{0} v_{1} \cdots v_{d}$ by attaching $n-d-2$ pendant edges to $v_{\lfloor d / 2\rfloor+1}$ and adding a new vertex $v_{d+1}$ to be adjacent to $v_{\lfloor d / 2\rfloor}$ and $v_{\lfloor d / 2\rfloor+1}$. The $n$ vertex graph arisen from $P_{d}=v_{0} v_{1} \cdots v_{d}$ by attaching $n-$ $d-3$ pendant edges to $v_{|d / 2|}$ and adding two new adjacent vertices $v_{d+1}$ and $v_{d+2}$ to be adjacent to $v_{\lfloor d / 2\rfloor}$, denoted by $\nabla_{n, d}$ (see Figure 2). So, if $d=2$, the graph $\nabla_{n, 2}$ arisen from $C_{3}$ by attaching $n-3$ pendant edges to one vertex of $C_{3}$.

Note that $n$-vertex graph $\triangle_{n, d}^{3}$ arisen from $P_{d}=v_{0} v_{1} \cdots v_{d}$ by attaching $n-d-3$ pendant edges to $v_{\lfloor d / 2\rfloor}$ and one pendant edge to $v_{\lfloor d / 2\rfloor+1}$ and adding a new vertex $v_{d+1}$ to be adjacent to $v_{\lfloor d / 2\rfloor}$ and $v_{\lfloor d / 2\rfloor+1} . \Delta_{n, d}^{4}$ arisen from $P_{d}=v_{0} v_{1} \cdots v_{d}$ by attaching $n-d-2$ pendant edges to $v_{\lceil d / 2\rceil}$ and adding a new vertex $v_{d+1}$ to be adjacent to $v_{\lceil d / 2\rceil}$ and $v_{\lceil d / 2\rceil+1}$.

Let $P_{d}=v_{0} v_{1} \cdots v_{d}, U_{0}\left(v_{i}\right)$ be the graph with $d+2$ vertices arisen from $P_{d}$ by adding a new vertex $u_{0}$ to be adjacent to one vertex $v_{i}\left(v_{i} \in V\left(P_{d}\right), 1 \leq i \leq d-1\right) . P_{d}\left(C_{3} ; v_{i}\right)$ arisen from $P_{d}=v_{0} v_{1} \cdots v_{d}$ by attaching $n-d-3$ pendant vertices to $v_{i}(1 \leq i \leq d-1)$ and identifying $v_{i}$ with one vertex of $C_{3}$, denote $U_{n, d}^{1}=\left\{P_{d}\left(C_{3} ; v_{i}\right) \mid 1 \leq i \leq d-1\right\}$. Let $U_{d+2}$ be a $(d+2)$ vertex unicyclic graph arisen from $P_{d}=v_{0} v_{1} \cdots v_{d}$ by adding a new vertex $v_{d+1}$ to be adjacent to $v_{k}$ and $v_{k+1}$ (see Figure 2), $U_{d+2}\left(n-d-2 ; v_{j}\right)$ arisen from $U_{d+2}$ by attaching $n-d-2$ 


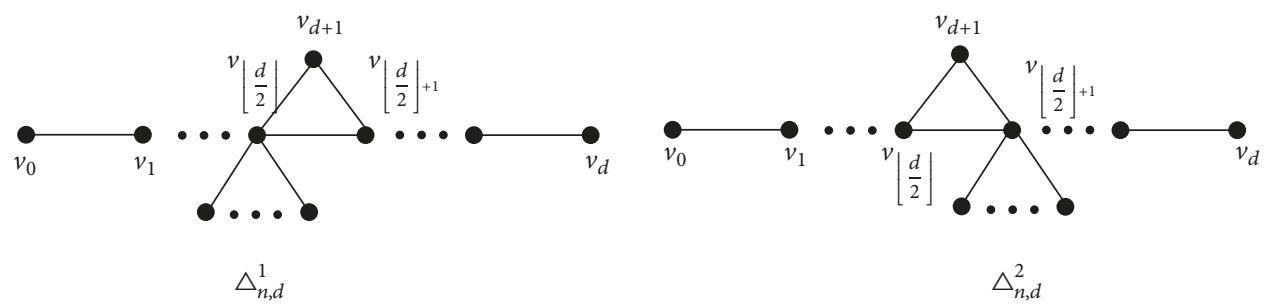

FIGURE 1: The graphs $\triangle_{n, d}^{1}$ and $\triangle_{n, d}^{2}$.

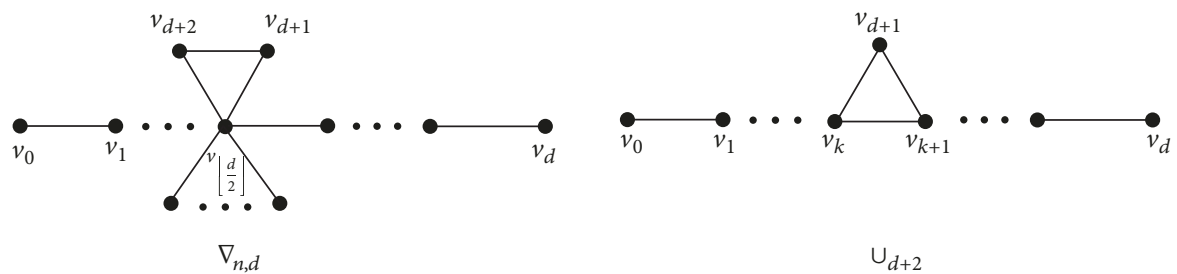

Figure 2: The graphs $\nabla_{n, d}$ and $U_{d+2}$.

pendant vertices to $v_{j}(1 \leq j \leq d-1$, or $j=d+1)$, and denote $U_{n, d}^{2}=\left\{U_{d+2}\left(n-d-2 ; v_{j}\right) \mid 1 \leq j \leq d-1\right.$, or $\left.j=d+1\right\}$.

Lately, the investigations on the spectral radius or eigenvalue or topological index of a special class of graphs and the topological properties of a certain class of networks remain a popular topic to researchers. For example, J.Q. Fei and J.H. Tu [4] characterized the complete characterization of the degree Kirchhoff index of bicyclic graphs. J.B. Liu et al. [5] discussed the topological properties of certain neural networks. B. Ning and $\mathrm{B}$. Li [6] discussed the spectral radius of connected clawfree graphs. Y.Y. Wu and Y.J. Chen [7] analyzed the eccentric connectivity index of graphs. C. Wang et al. [8] obtained the least eigenvalue of the graphs whose complements are connected and have pendant paths. Many results can be seen in [2-16] and other papers. In particular, A. Ilic et al. [13] discussed the Harary index among the trees with fixed diameter. In this paper, we determine the graphs which have the maximum and second-maximum Harary indices among all the $n$-vertex unicyclic graphs with diameter $d$.

\section{Lemmas}

Lemma 1 (see [14]). Let $A, H_{1}$, and $H_{2}$ be three connected, pairwise disjoint graphs; note that $u_{1}, u_{2} \in V(A), v_{1} \in V\left(H_{1}\right)$, $w_{1} \in V\left(H_{2}\right)$. The graph $G$ is obtained by identifying the vertices $u_{1}, v_{1}$ and $u_{2}, w_{1}$ between $A, H_{1}, H_{2}$, respectively. $G^{\prime}$ is obtained by identifying the vertices $u_{1}, v_{1}, w_{1}$ from $A, H_{1}, H_{2}$ and $G^{\prime \prime}$ is obtained by identifying the vertices $u_{2}, v_{1}, w_{1}$ from $A, H_{1}, H_{2}$; then we can get

$$
\begin{aligned}
& H(G)<H\left(G^{\prime}\right) \text { or } \\
& H(G)<H\left(G^{\prime \prime}\right) .
\end{aligned}
$$

Lemma 2 (see [9]). Let $G_{1}$ be an n-vertex unicyclic graph and $\nabla_{n, 2}$ be the graph defined as above. Then

$$
H\left(G_{1}\right) \leq H\left(\nabla_{n, 2}\right),
$$

if and only if $G_{1} \cong \nabla_{n, 2}$, and the equality holds. When $d=1$, then $G_{1} \cong C_{3}$, and when $d=2, n \leq 5$, then $G_{1} \cong C_{4}$.

Lemma 3. Let $U_{0}\left(v_{i}\right)$ be the graph defined as above; then

$$
H\left(U_{0}\left(v_{\lfloor d / 2\rfloor}\right)\right) \geq H\left(U_{0}\left(v_{i}\right)\right)
$$

if and only if $i=\lfloor d / 2\rfloor$, the equality holds.

Proof. By the calculation of Harary index, we can get the following.

(1) If $1 \leq i<\lfloor d / 2\rfloor$,

$$
\begin{aligned}
& H\left(U_{0}\left(v_{i+1}\right)\right)-H\left(U_{0}\left(v_{i}\right)\right)=\left(\frac{1}{i+2}-\frac{1}{d-i+1}\right) \\
& \quad>0 .
\end{aligned}
$$

(2) If $\lfloor d / 2\rfloor<i \leq d-1$,

$$
\begin{aligned}
& H\left(U_{0}\left(v_{i-1}\right)\right)-H\left(U_{0}\left(v_{i}\right)\right)=\left(\frac{1}{d-i+2}-\frac{1}{i+1}\right) \\
& \quad>0 .
\end{aligned}
$$

When $d$ is odd, we can get $H\left(U_{0}\left(v_{\lfloor d / 2\rfloor}\right)\right)=$ $H\left(U_{0}\left(v_{\lfloor d / 2\rfloor+1}\right)\right)$.

Thus the result holds.

Lemma 4. Let $U_{n, d}^{2}=\left\{U_{d+2}\left(n-d-2 ; v_{j}\right) \mid 1 \leq j \leq d-\right.$ 1 , or $j=d+1\}$ and $\triangle_{n, d}^{1}$ and $\triangle_{n, d}^{2}$ be the set and two graphs defined as above, respectively. $G_{1} \in U_{n, d}^{2}$; then

$$
H\left(G_{1}\right) \leq H\left(\triangle_{n, d}^{1}\right)
$$

if and only if $G_{1} \cong \triangle_{n, d}^{1}$, the equality holds. 
Proof. Choose a graph $G_{1} \in U_{n, d}^{2}(3 \leq d \leq n-2)$, such that $H\left(G_{1}\right)$ is the maximum. The following claims play a crucial role.

Claim 1. $j \in\{k, k+1,\lfloor d / 2\rfloor,\lfloor d / 2\rfloor+1\}$.

Proof. First, we prove $j \neq d+1$. Otherwise, $j=d+1$; denote $u_{i} \in N_{G_{1}}\left(v_{d+1}\right) \backslash\left\{v_{k}, v_{k+1}\right\}, 1 \leq i \leq n-d-2$; let

$$
\begin{aligned}
& G_{1}^{*}=G_{1}-\sum_{i=1}^{n-d-2} v_{d+1} u_{i}+\sum_{i=1}^{n-d-2} v_{k} u_{i} \text { or } \\
& G_{1}^{*}=G_{1}-\sum_{i=1}^{n-d-2} v_{d+1} u_{i}+\sum_{i=1}^{n-d-2} v_{k+1} u_{i} ;
\end{aligned}
$$

using Lemma 1 , we get $H\left(G_{1}\right)<H\left(G_{1}^{*}\right)$, a contraction.

So, $1 \leq j \leq d-1$, let $w_{i} \in N_{G_{1}}\left(v_{j}\right) \backslash\left\{v_{j-1}, v_{j+1}\right\}, 1 \leq i \leq$ $n-d-2, N_{P_{d}}\left(v_{j}\right)=\left\{v_{j-1}, v_{j+1}\right\}$.

(1) If $1 \leq j<k \leq\lfloor d / 2\rfloor$, let

$$
G_{1}^{*}=G_{1}-\sum_{i=1}^{n-d-2} v_{j} w_{i}+\sum_{i=1}^{n-d-2} v_{k} w_{i} ;
$$

if $\lfloor d / 2\rfloor \leq k<k+1<j \leq d-1$, let

$$
G_{1}^{*}=G_{1}-\sum_{i=1}^{n-d-2} v_{j} w_{i}+\sum_{i=1}^{n-d-2} v_{k+1} w_{i} .
$$
let

(2) If $1 \leq k+1<j \leq\lfloor d / 2\rfloor$ or $\lfloor d / 2\rfloor \leq j<k<k+1 \leq d-1$,

$$
G_{1}^{*}=G_{1}-v_{d+1} v_{k}-v_{d+1} v_{k+1}+v_{d+1} v_{j}+v_{d+1} v_{j+1} .
$$

(3) If $1 \leq j<\lfloor d / 2\rfloor<k, k+1 \leq d-1$, let

$$
\begin{aligned}
G_{1}^{*}= & G_{1}-\sum_{i=1}^{n-d-2} v_{j} w_{i}-v_{d+1} v_{k}-v_{d+1} v_{k+1} \\
& +\sum_{i=1}^{n-d-2} v_{\lfloor d / 2\rfloor} w_{i}+v_{d+1} v_{\lfloor d / 2\rfloor}+v_{d+1} v_{\lfloor d / 2\rfloor+1} ;
\end{aligned}
$$

i.e., $G_{1}^{*} \cong \triangle_{n, d}^{1}$;

if $1 \leq k<k+1<\lfloor d / 2\rfloor+1<j \leq d-1$, let

$$
\begin{aligned}
G_{1}^{*}= & G_{1}-\sum_{i=1}^{n-d-2} v_{j} w_{i}-v_{d+1} v_{k}-v_{d+1} v_{k+1} \\
& +\sum_{i=1}^{n-d-2} v_{\lfloor d / 2\rfloor+1} w_{i}+v_{d+1} v_{\lfloor d / 2\rfloor}+v_{d+1} v_{\lfloor d / 2\rfloor+1} ;
\end{aligned}
$$

i.e., $G_{1}^{*} \cong \triangle_{n, d}^{2}$.

From Lemma 3 and the calculation of $H\left(G_{1}\right)$, we can calculate that $H\left(G_{1}\right)<H\left(G_{1}^{*}\right)$, a contraction.

Claim 2. $k=\lfloor d / 2\rfloor$.

Proof. Otherwise, if $k=j<\lfloor d / 2\rfloor$, let $G_{1}^{*}=G_{1}-v_{d-1} v_{d}+v_{0} v_{d}$; if $k=j>\lfloor d / 2\rfloor$, let $G_{1}^{*}=G_{1}-v_{0} v_{1}+v_{0} v_{d}$. From Lemma 3, we can get $H\left(G_{1}\right)<H\left(G_{1}^{*}\right)$, a contraction.
By Claims 1-2, we have the graph $G_{1} \cong \triangle_{n, d}^{1}$ or $G_{1} \cong \triangle_{n, d}^{2}$.

Claim 3. $H\left(\triangle_{n, d}^{2}\right) \leq H\left(\triangle_{n, d}^{1}\right)$.

Proof. If $d=2 t$ is even, we have

$$
\begin{aligned}
& H\left(\Delta_{n, d}^{2}\right)-H\left(\triangle_{n, d}^{1}\right)=H\left(\Delta_{n, 2 t}^{2}\right)-H\left(\Delta_{n, 2 t}^{1}\right)=(n \\
& -2 t-2)\left\{\left(1+\frac{1}{t+1}+\frac{1}{t+2}+2 \sum_{i=2}^{t} \frac{1}{i}\right)\right. \\
& \left.-\left(1+2 \sum_{i=2}^{t+1} \frac{1}{i}\right)\right\}=(n-2 t-2)\left(\frac{1}{t+2}-\frac{1}{t+1}\right)
\end{aligned}
$$

0 .

$<0$.

If $d=2 t+1$ is odd, $\triangle_{n, 2 t+1}^{1} \cong \triangle_{n, 2 t+1}^{2}, H\left(\triangle_{n, d}^{1}\right)-H\left(\triangle_{n, d}^{2}\right)=$

By Claims 1-3, we have $G_{1} \cong \triangle_{n, d}^{1}$.

Thus the result follows.

\section{Main Results}

In this section, we will list our main results.

Theorem 5. Let $U_{n, d}$ and $\triangle_{n, d}^{1}$ be defined in Section 1, the nvertex graph $G_{1} \in U_{n, d}(3 \leq d \leq n-2)$; then

$$
H\left(\triangle_{n, d}^{1}\right) \geq H\left(G_{1}\right)
$$

if and only if $G_{1} \cong \triangle_{n, d}^{1}$, the equality holds.

Proof. Let $G_{1} \in U_{n, d}$; using Lemma 2, the result holds for $d=$ 1,2 . If $d=n-1$, then $G_{1} \cong C_{n}$. So, we discuss that $3 \leq d \leq$ $n-2$.

Choose a graph $G_{1} \in U_{n, d}$ with $H\left(G_{1}\right)$ being the maximum. Note that $C_{q}$ is the only cycle and $P_{d}=v_{0} v_{1} \cdots v_{d}$ is the induced path in $G_{1}$; assume that $d\left(v_{0}\right)=1$; we consider the following claims.

Claim 1. $\left|V\left(C_{q}\right) \cap V\left(P_{d}\right)\right|>0$.

Proof. Otherwise, suppose that there exists a path $P_{k}=$ $v_{h} v_{g+1} \cdots v_{g+k-1} v_{l}$ connecting the cycle $C_{q}$ and the path $P_{d}$ with $v_{h} \in V\left(P_{d}\right), v_{l} \in V\left(C_{q}\right)$; denote $u_{s} \in N_{G_{1}}\left(v_{h}\right) \backslash\left\{v_{g+1}\right\}, 1 \leq$ $s \leq d\left(v_{h}\right)-1$ and $w_{j} \in N_{G_{1}}\left(v_{l}\right) \backslash\left\{v_{g+k-1}\right\}, 1 \leq j \leq d\left(v_{l}\right)-1$. Let

$$
\begin{aligned}
& G_{1}^{*}=G_{1}-\sum_{s=1}^{d\left(v_{h}\right)-1} v_{h} u_{s}+\sum_{s=1}^{d\left(v_{h}\right)-1} v_{l} u_{s} \text { or } \\
& G_{1}^{*}=G_{1}-\sum_{j=1}^{d\left(v_{l}\right)-1} v_{l} w_{j}+\sum_{j=1}^{d\left(v_{l}\right)-1} v_{h} w_{j} .
\end{aligned}
$$

Then, applying Lemma $1, H\left(G_{1}\right)<H\left(G_{1}^{*}\right)$, a contraction. 
Using Claim 1, we note that $V\left(C_{q}\right) \cap V\left(P_{d}\right)=\left\{v_{t}, v_{t+1}, \cdots\right.$, $\left.v_{t+k}\right\}, V\left(C_{q}\right) \backslash V\left(P_{d}\right)=\left\{v_{d+1}, v_{d+2}, \cdots, v_{s}\right\}$.

Claim 2. For any $v \in V\left(G_{1}\right) \backslash\left(V\left(P_{d}\right) \bigcup V\left(C_{q}\right)\right), d(v)=1$.

Proof. Otherwise, assume that there exists a vertex $u \in V\left(G_{1}\right) \backslash$ $\left(V\left(P_{d}\right) \cup V\left(C_{q}\right)\right)$ with $u v_{b}(1 \leq b \leq d(u)-1), u v_{f} \in E\left(G_{1}\right)$, $v_{b} \in V\left(G_{1}\right) \backslash\left(V\left(P_{d}\right) \cup V\left(C_{q}\right)\right), v_{f} \in V\left(P_{d}\right) \bigcup V\left(C_{q}\right)$. Suppose that $u_{s}^{\prime} \in N_{G_{1}}\left(v_{f}\right) \backslash\{u\}, 1 \leq s \leq d\left(v_{f}\right)-1$. Let

$$
\begin{aligned}
& G_{1}^{*}=G_{1}-\sum_{s=1}^{d\left(v_{f}\right)-1} v_{f} u_{s}^{\prime}+\sum_{s=1}^{d\left(v_{f}\right)-1} u u_{s}^{\prime} \text { or } \\
& G_{1}^{*}=G_{1}-\sum_{b=1}^{d(u)-1} v_{b} u+\sum_{b=1}^{d(u)-1} v_{b} v_{f} .
\end{aligned}
$$

Then, using Lemma $1, H\left(G_{1}\right)<H\left(G_{1}^{*}\right)$, a contraction.

By Claim 2, we have any vertex $v \in V\left(G_{1}\right) \backslash\left(V\left(P_{d}\right) \bigcup\right.$ $\left.V\left(C_{q}\right)\right)$ is pendant vertex.

Claim 3. $\left|V\left(C_{q}\right) \cap V\left(P_{d}\right)\right| \leq 2$.

Proof. Otherwise, suppose that $V\left(C_{q}\right) \cap V\left(P_{d}\right)=\left\{v_{t}, v_{t+1}, \cdots\right.$, $\left.v_{t+k}\right\}, k \geq 2$.

(1) If $t \leq\lfloor d / 2\rfloor-1$, denote $u_{i} \in N_{G_{1}}\left(v_{t}\right) \backslash\left\{v_{s}, v_{t-1}, v_{t+1}\right\}$, $1 \leq i \leq d\left(v_{t}\right)-3$ (if exists), $N_{P_{d}}\left(v_{t}\right)=\left\{v_{t-1}, v_{t+1}\right\}$. Let

$$
G_{1}^{*}=G_{1}-v_{s} v_{t}+v_{s} v_{t+1}-\sum_{i=1}^{d\left(v_{t}\right)-3} v_{t} u_{i}+\sum_{i=1}^{d\left(v_{t}\right)-3} v_{t+1} u_{i} .
$$

(2) If $t+k \geq\lfloor d / 2\rfloor+2$, denote $u_{s} \in N_{G_{1}}\left(v_{t+k}\right) \backslash\left\{v_{d+1}\right.$, $\left.v_{t+k-1}, v_{t+k+1}\right\}, 1 \leq s \leq d\left(v_{t+k}\right)-3$ (if exists), $N_{P_{d}}\left(v_{t+k}\right)=$ $\left\{v_{t+k-1}, v_{t+k+1}\right\}$. Let

$$
\begin{aligned}
G_{1}^{*}= & G_{1}-v_{d+1} v_{t+k}+v_{d+1} v_{t+k-1}-\sum_{s=1}^{d\left(v_{t+k}\right)-3} v_{t+k} u_{s} \\
& +\sum_{s=1}^{d\left(v_{t+k}\right)-3} v_{t+k-1} u_{s} .
\end{aligned}
$$

Then, applying Lemma 3 and the calculation of $H\left(G_{1}\right)$, $H\left(G_{1}\right)<H\left(G_{1}^{*}\right)$, a contraction.

Claim 4. (1) if $\left|V\left(C_{q}\right) \cap V\left(P_{d}\right)\right|=2, s=d+1$; (2) if $\mid V\left(C_{q}\right) \cap$ $V\left(P_{d}\right) \mid=1, s=d+2$.

Proof. (1) Otherwise, we assume that $s \geq d+2$. The graph $G_{1}^{*}$ arisen by deleting all edges in $E\left(C_{q}\right)$ of $G_{1}$ and incidence with $C_{q}$ except the edges $v_{t} v_{t+1}, v_{t+1} v_{d+1}$ of $G_{1}$, adding the edge $v_{t} v_{d+1}$, connecting all isolated vertices to the vertex $v_{t}$ (if $t \geq\lfloor d / 2\rfloor$ ) or to the vertex $v_{t+1}$ (if $t+1 \leq\lfloor d / 2\rfloor$ ) of $G_{1}$. Together with the definition of the Harary index and Lemma 3, $H\left(G_{1}\right)<H\left(G_{1}^{*}\right)$, a contraction.

(2) Otherwise, we assume that $s \geq d+3$. The graph $G_{1}^{*}$ is obtained from $G_{1}$ by deleting all edges in $E\left(C_{q}\right)$ and incidence with $C_{q}$ except the edges $v_{t} v_{d+1}, v_{d+1} v_{d+2}$, adding the edge $v_{t} v_{d+2}$, connecting all isolated vertices to the vertex $v_{t}$. We can calculate that $H\left(G_{1}\right)<H\left(G_{1}^{*}\right)$, a contraction.
By Claim 4 and Lemmas 1 and 3, we get $G_{1} \in U_{n, d}^{1} \cup U_{n, d}^{2}$. If $G_{1} \in U_{n, d}^{1}$, applying Lemma $3, G_{1} \cong \nabla_{n, d}$ (see Figure 2); if $G_{1} \in U_{n, d}^{2}$, applying Lemma $4, G_{1} \cong \triangle_{n, d}^{1}$.

Claim 5. $H\left(\triangle_{n, d}^{1}\right)>H\left(\nabla_{n, d}\right)$.

Proof. If $d=2 t$ is even, we have

$$
\begin{aligned}
H & \left(\Delta_{n, d}^{1}\right)-H\left(\nabla_{n, d}\right)=H\left(\triangle_{n, 2 t}^{1}\right)-H\left(\nabla_{n, 2 t}\right) \\
& =\left\{\left(\frac{1}{t+1}+2 \sum_{i=1}^{t} \frac{1}{i}+\frac{1}{2}\right)-\left(1+2 \sum_{i=2}^{t+1} \frac{1}{i}+1\right)\right\} \\
& =\frac{1}{2}-\frac{1}{t+1}>0 .
\end{aligned}
$$

If $d=2 t+1$ is odd, we have

$$
\begin{aligned}
H & \left(\triangle_{n, d}^{1}\right)-H\left(\nabla_{n, d}\right)=H\left(\Delta_{n, 2 t+1}^{1}\right)-H\left(\nabla_{n, 2 t+1}\right) \\
& =\left\{\left(2 \sum_{i=1}^{t+1} \frac{1}{i}+\frac{1}{2}\right)-\left(1+2 \sum_{i=2}^{t+1} \frac{1}{i}+\frac{1}{t+2}+1\right)\right\} \\
& =\frac{1}{2}-\frac{1}{t+2}>0 .
\end{aligned}
$$

By Claims 1-5, we have $G_{1} \cong \triangle_{n, d}^{1}$.

Thus the result follows.

Theorem 6. Let $U_{n, d}, \triangle_{n, d}^{1}$ be defined in Section 1; the graph $G_{1} \in U_{n, d} \backslash\left\{\Delta_{n, d}^{1}\right\}(3 \leq d \leq n-2)$; then

(1) If $d=2 t$ is even, we obtain

$$
H\left(\nabla_{n, d}\right) \geq H\left(G_{1}\right), \quad \text { when } 2 n \geq t^{2}+5 t+2 ;
$$

if and only if $G_{1} \cong \nabla_{n, d}$, the equality holds.

$$
H\left(\triangle_{n, d}^{2}\right) \geq H\left(G_{1}\right)
$$

when $4 t+8 \leq 2 n \leq t^{2}+5 t+2$;

if and only if $G_{1} \cong \triangle_{n, d}^{2}$, the equality holds.

(2) If $d=2 t+1$ is odd, we have

$$
H\left(\triangle_{n, d}^{4}\right) \geq H\left(G_{1}\right)
$$

if and only if $G_{1} \cong \triangle_{n, d}^{4}$, the equality holds.

Proof. Choose a graph $G_{1} \in U_{n, d} \backslash\left\{\triangle_{n, d}^{1}\right\}$, such that $H\left(G_{1}\right)$ is as large as possible; together with Lemmas 1,3 , and 4 and Theorem 5 , we have the following.

(1) If $d=2 t$ is even, $G_{1} \cong \triangle_{n, d}^{2}$ or $G_{1} \cong \triangle_{n, d}^{3}$ or $G_{1} \cong \nabla_{n, d}$. 
In fact,

$$
\begin{aligned}
& H\left(\triangle_{n, d}^{3}\right)-H\left(\triangle_{n, d}^{2}\right) \\
& =(n-d-3)\left(\frac{11}{6}+2 \sum_{i=2}^{t+1} \frac{1}{i}\right) \\
& -(n-d-3)\left(2+2 \sum_{i=2}^{t} \frac{1}{i}+\frac{1}{t+1}+\frac{1}{t+2}\right) \\
& =(n-d-3)\left(\frac{1}{t+1}-\frac{1}{t+2}-\frac{1}{6}\right)<0, \\
& H\left(\triangle_{n, d}^{3}\right)-H\left(\nabla_{n, d}\right) \\
& =\left(\frac{3}{2}+2 \sum_{i=2}^{t} \frac{1}{i}+\frac{1}{t+1}+\frac{1}{t+2}+\frac{n-d-3}{3}\right) \\
& -\left(2+2 \sum_{i=2}^{t+1} \frac{1}{i}+\frac{n-d-3}{2}\right) \\
& \left.=-\frac{1}{(t+1)(t+2)}-\frac{1}{2}-\frac{n-d-3}{6}\right)<0, \\
& H\left(\nabla_{n, d}\right)-H\left(\triangle_{n, d}^{2}\right) \\
& =(n-d-3)\left(2+2 \sum_{i=2}^{t+1} \frac{1}{i}\right) \\
& -(n-d-3)\left(2+2 \sum_{i=2}^{t} \frac{1}{i}+\frac{1}{t+1}+\frac{1}{t+2}\right) \\
& +\left(3+4 \sum_{i=2}^{t+1} \frac{1}{i}\right) \\
& -\left(\frac{7}{2}+4 \sum_{i=2}^{t} \frac{1}{i}+\frac{2}{t+1}+\frac{1}{t+2}\right) \\
& =(n-d-2)\left(\frac{1}{t+1}-\frac{1}{t+2}\right)+\left(\frac{1}{t+1}-\frac{1}{2}\right) \text {. }
\end{aligned}
$$

(1.1) When $2 n \geq t^{2}+5 t+2, H\left(\nabla_{n, d}\right)-H\left(\triangle_{n, d}^{2}\right) \geq 0$.

(1.2) When $4 t+8 \leq 2 n \leq t^{2}+5 t+2, H\left(\nabla_{n, d}\right)-H\left(\Delta_{n, d}^{2}\right) \leq 0$.

(2) If $d=2 t+1$ is odd, $H\left(\triangle_{n, d}^{1}\right)=H\left(\triangle_{n, d}^{2}\right)$, so $G_{1} \cong \triangle_{n, d}^{3}$ or $G_{1} \cong \triangle_{n, d}^{4}$ or $G_{1} \cong \nabla_{n, d}$.

$$
\begin{aligned}
H\left(\triangle_{n, d}^{3}\right)-H\left(\triangle_{n, d}^{4}\right) \\
=\frac{1}{3}(n-d-3)-\frac{1}{2}(n-d-3) \\
\quad+\left(3+\frac{1}{t+2}+4 \sum_{i=2}^{t+1} \frac{1}{i}\right) \\
\quad-\left(3+\frac{2}{t+2}+3 \sum_{i=2}^{t+1} \frac{1}{i}+\sum_{i=2}^{t} \frac{1}{i}\right)
\end{aligned}
$$

$$
\begin{aligned}
= & (n-d-3)\left(\frac{1}{3}-\frac{1}{2}\right)+\left(\frac{1}{t+1}-\frac{1}{t+2}\right)<0, \\
H\left(\nabla_{n, d}\right)-H\left(\triangle_{n, d}^{4}\right) & \\
= & \left(3+\frac{2}{t+2}+4 \sum_{i=2}^{t+1} \frac{1}{i}\right) \\
& -\left(\frac{7}{2}+\frac{2}{t+2}+3 \sum_{i=2}^{t+1} \frac{1}{i}+\sum_{i=2}^{t} \frac{1}{i}\right) \\
= & \left(\frac{1}{t+1}-\frac{1}{2}\right)<0 .
\end{aligned}
$$

Thus the result follows.

\section{Data Availability}

The data used to support the findings of this study are included within the article.

\section{Conflicts of Interest}

The authors declare that they have no conflicts of interest.

\section{Acknowledgments}

This paper is supported by the Natural Science Foundation of China (11871077), the National Natural Science Foundation of China (11371028), the Natural Science Foundation of Anhui Province of Anhui (1808085MA04).

\section{References}

[1] J. A. Bondy and U. S. R. Murty, Graph Theory with Application, Macmillan Press, New York, NY, USA, 1976.

[2] O. Ivanciuc, T.-S. Balaban, and A. T. Balaban, "Design of topological indices. Part 4. Reciprocal distance matrix, related local vertex invariants and topological indices," Journal of Mathematical Chemistry, vol. 12, no. 1-4, pp. 309-318, 1993.

[3] D. Plavšić, S. Nikolić, N. Trinajstić, and Z. Mihalić, "On the Harary index for the characterization of chemical graphs," Journal of Mathematical Chemistry, vol. 12, no. 1-4, pp. 235-250, 1993.

[4] J. Fei and J. Tu, "Complete characterization of bicyclic graphs with the maximum and second-maximum degree Kirchhoff index," Applied Mathematics and Computation, vol. 330, pp. 118124,2018

[5] J. Liu, J. Zhao, S. Wang, M. Javaid, and J. Cao, "On the Topological Properties of the Certain Neural Networks," Journal of Artificial Intelligence and Soft Computing Research, vol. 8, no. 4, pp. 257-268, 2018.

[6] B. Ning and B. Li, "Spectral radius and traceability of connected claw-free graphs," Filomat, vol. 30, pp. 2445-2452, 2016.

[7] Y. Wu and Y. Chen, "On the extremal eccentric connectivity index of graphs," Applied Mathematics and Computation, vol. 331, pp. 61-68, 2018. 
[8] C. Wang, G. Yu, W. Sun, and J. Cao, "The Least Eigenvalue of the Graphs Whose Complements Are Connected and Have Pendent Paths," Journal of Artificial Intelligence and Soft Computing Research, vol. 8, no. 4, pp. 303-308, 2018.

[9] D. Chen, The Harary index of a unicyclic graph [M.S. thesis], Hunan Normal University, Changsha, China, 2009.

[10] K. C. Das, B. Zhou, and N. Trinajstic, "Bounds on Harary index," Journal of Mathematical Chemistry, vol. 46, no. 4, pp. 1377-1393, 2009.

[11] L. Feng and A. Ilić, "Zagreb, Harary and hyper-Wiener indices of graphs with a given matching number," Applied Mathematics Letters, vol. 23, no. 8, pp. 943-948, 2010.

[12] S. He and S. Li, "On the signless Laplacian index of unicyclic graphs with fixed diameter," Linear Algebra and its Applications, vol. 436, no. 1, pp. 252-261, 2012.

[13] A. Ilic, G. Yu, and L. Feng, “The Harary index of trees," Utilitas Mathematica, vol. 87, pp. 21-31, 2012.

[14] K. Xu and N. Trinajstić, "Hyper-Wiener and Harary indices of graphs with cut edges," Utilitas Mathematica, vol. 84, pp. 153163, 2011.

[15] K. Xu and K. C. Das, "Extremal unicyclic and bicyclic graphs with respect to Harary index," Bulletin of the Malaysian Mathematical Sciences Society, vol. 36, no. 2, pp. 373-383, 2013.

[16] Y. Nacaroglu and A. D. Maden, "On the eccentric connectivity index of unicyclic graphs," Iranian Journal of Mathematical Chemistry, vol. 9, pp. 47-56, 2018. 


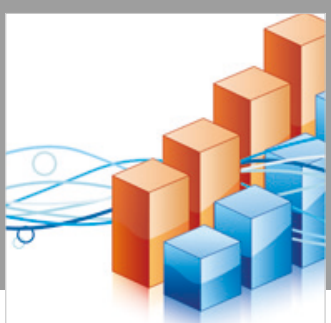

Advances in

Operations Research

\section{-n-m}
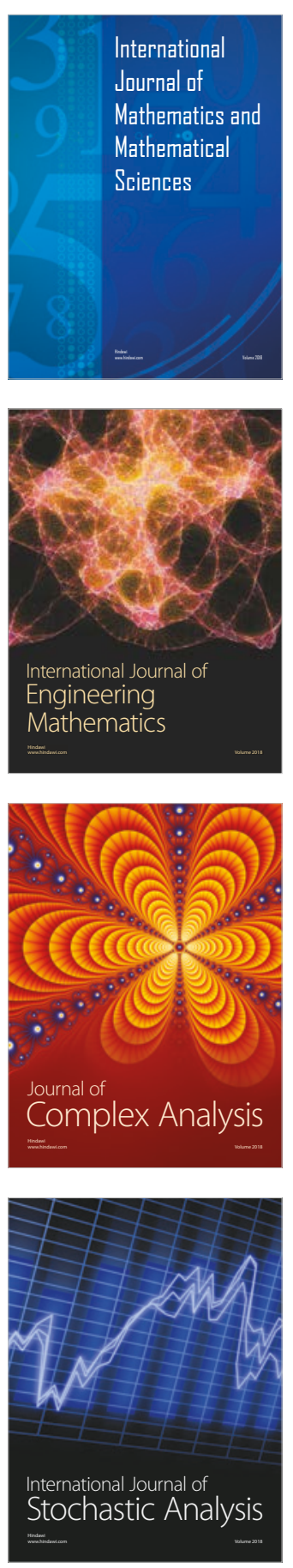
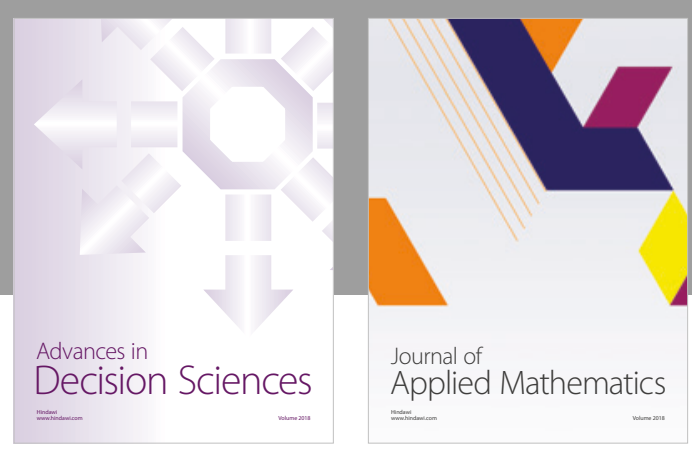

Journal of

Applied Mathematics
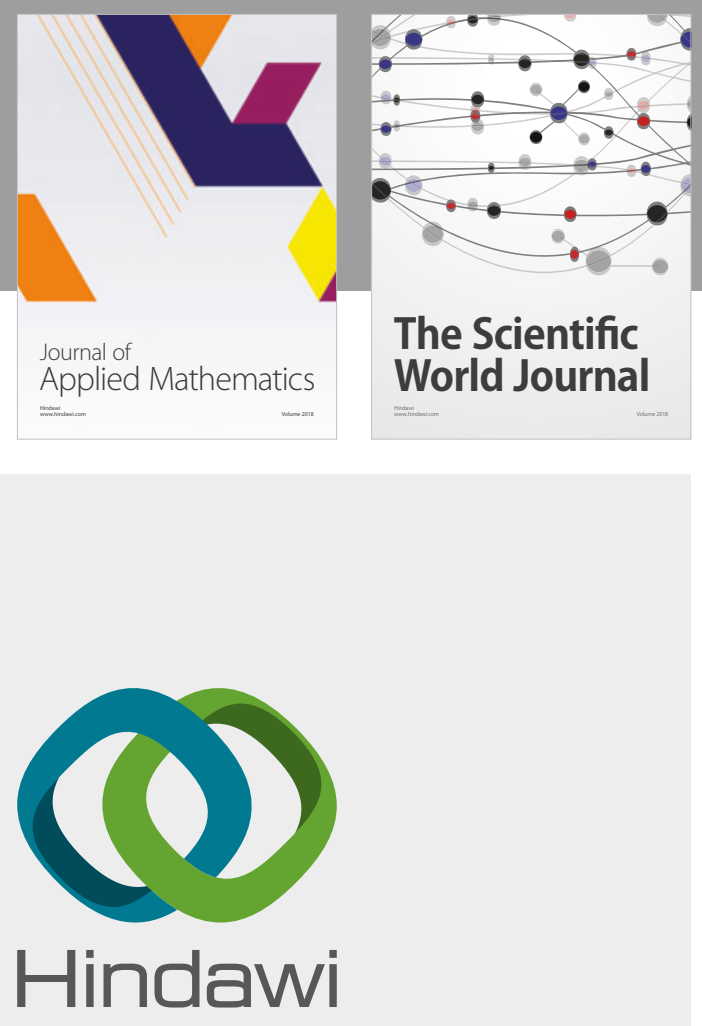

Submit your manuscripts at

www.hindawi.com

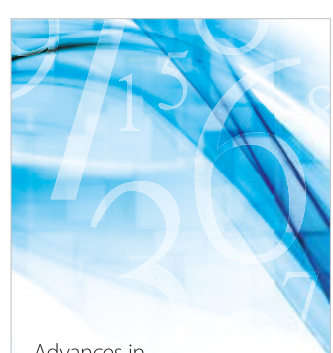

Advances in
Numerical Analysis
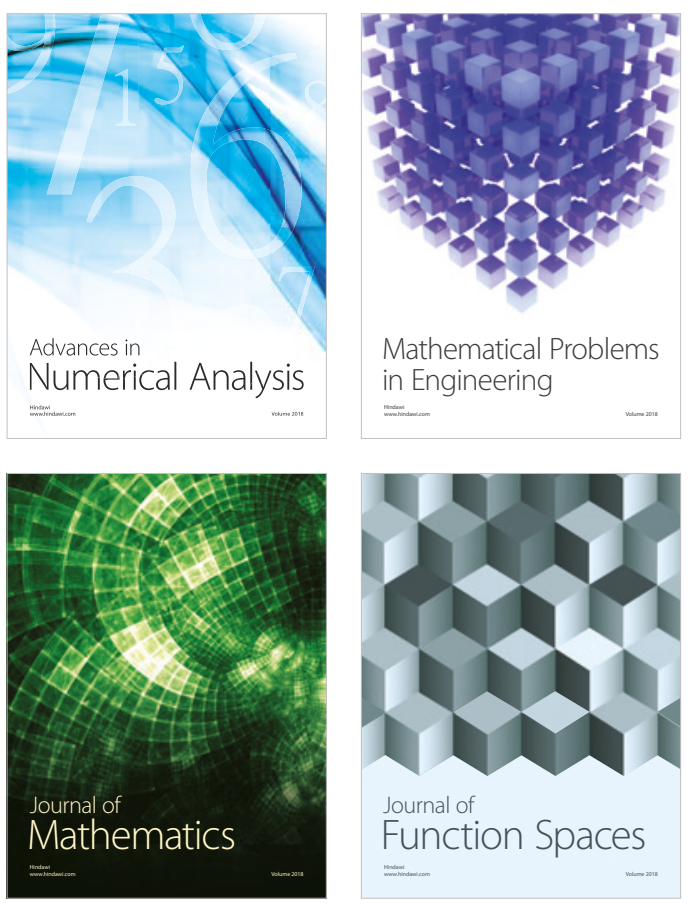

Mathematical Problems in Engineering

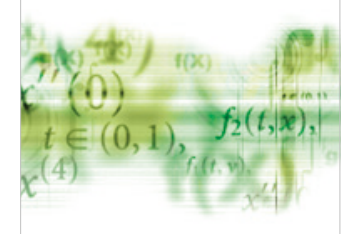

International Journal of

Differential Equations

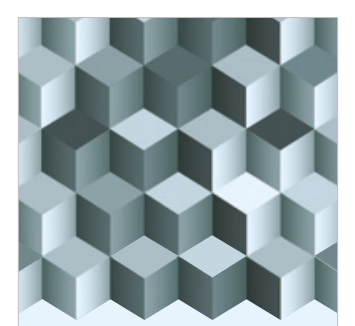

Journal of

Function Spaces

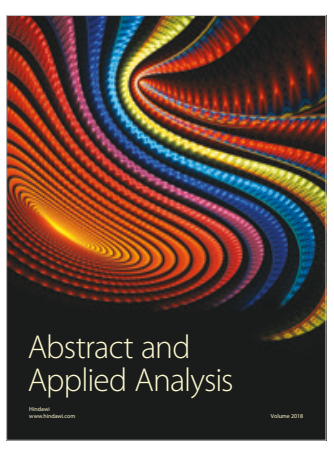

The Scientific

World Journal

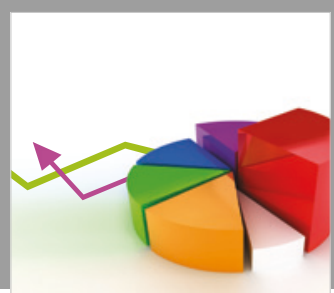

Journal of

Probability and Statistics
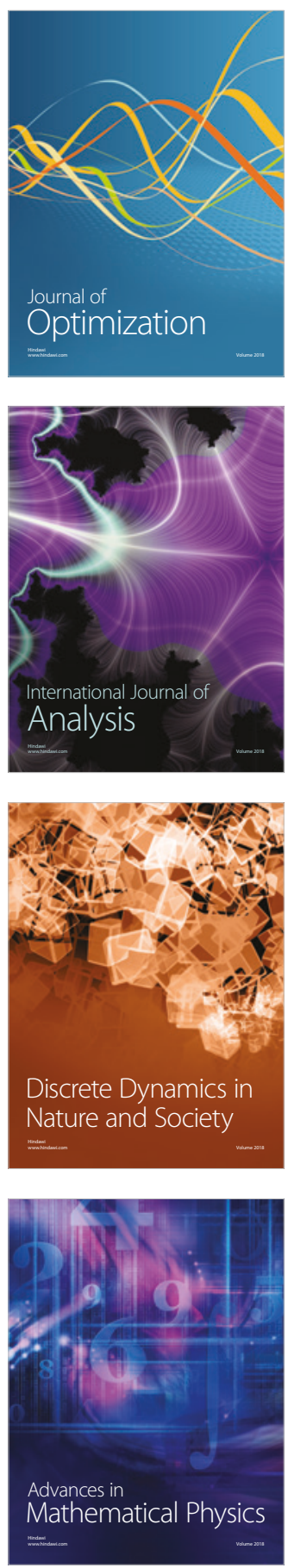